\title{
Full-field drift Hamiltonian particle orbits in axisymmetric tokamak geometry
}

\author{
W. A. Cooper, ${ }^{1, a)}$ G. A. Cooper, ${ }^{1}$ J. P. Graves, ${ }^{1}$ and M. Yu. Isaev ${ }^{2}$ \\ ${ }^{1}$ Ecole Polytechnique Fédérale de Lausanne, Centre de Recherches en Physique des Plasmas, \\ Association Euratom-Confédération Suisse, CH1015 Lausanne, Switzerland \\ ${ }^{2}$ Russian Research Centre “Kurchatov Institute," 123182 Moscow, Russia
}

(Received 23 December 2010; accepted 28 March 2011; published online 27 May 2011)

\begin{abstract}
A Hamiltonian/Lagrangian theory to describe guiding center orbit drift motion that is canonical in Boozer magnetic coordinates is developed to include full electrostatic and electromagnetic perturbed fields in axisymmetric tokamak geometry. Furthermore, the radial component of the equilibrium magnetic field in the covariant representation is retained and the background equilibrium state extends to anisotropic plasma pressure conditions. A gauge transformation on the perturbed vector potential is imposed to guarantee canonical structure in the Boozer frame. Perturbed field nonlinear wave-wave interactions affect only the evolution of the guiding center particle parallel gyroradius. The evolution of the particle coordinate positions retains only linear wave-particle interactions. For particle motion in magnetohydrodynamic (MHD) instability structures, the electrostatic potential is linked mainly to the binormal component of the perturbed displacement vector when finite $\delta \mathbf{A}_{\perp}$ components are included. (C) 2011 American Institute of Physics. [doi:10.1063/1.3579398]
\end{abstract}

\section{INTRODUCTION}

The tools of nonlinear Hamiltonian dynamics can greatly facilitate the implementation of numerical schemes for guiding center particle orbit motion when the coordinate system is canonical. ${ }^{1,2}$ The application of Lagrangian theory to identify the canonical momenta and angular variables constitutes a very valuable step. ${ }^{3}$ Previously, a canonical coordinate system for guiding center motion in arbitrary fields was devised, ${ }^{4}$ but its use is impractical. This is due to problems associated with the transformation from a coordinate system typically employed in magnetohydrodynamic (MHD) equilibrium and stability solvers to it. ${ }^{5}$ One approach that has allowed the inclusion of arbitrary fields but eliminates the radial component of the equilibrium magnetic field in the covariant representation is achieved by a redefinition of the guiding center drift velocity. ${ }^{6,7}$ An extension of this formulation to anisotropic pressure and three-dimensional (3D) geometry has been recently reported. ${ }^{8}$ A drift Hamiltonian formalism in generalized nonstraight field line coordinates has been described that is exact in axisymmetric systems and approximate in 3D geometry. ${ }^{9}$ A canonical drift Hamiltonian formulation in Boozer coordinates, which retains full stationary axisymmetric fields without selectively neglecting higher order terms, ${ }^{10}$ has been extended to include electrostatic and electromagnetic perturbations with only a finite parallel component of the vector potential. ${ }^{11}$ This last work also allowed for anisotropic pressure which can be very relevant for plasmas that are heated with auxiliary methods. ${ }^{12-17}$

In particular, we extend the formalism adopted in Ref. 11 to include the finite $\delta \mathbf{A}_{\perp}$ contribution to the guiding center drift orbit evolution equations in the article. In Sec. II, we specifically include the anisotropic pressure through the covariant representation of the equilibrium magnetic field

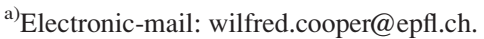

which can be interpreted as altering the permeability of the plasma. Finite electrostatic and full electromagnetic perturbed fields are considered in axisymmetric tokamak geometry. A set of canonical variables applicable to guiding center motion is defined through Lagrangian manipulations. The imposition of a specific gauge condition for the perturbed vector potential is required. The canonical variables form the basis of a Hamiltonian formulation of the equations of motion, which is presented in Sec. III. A transformation of canonical momenta to the Boozer radial coordinate and the parallel gyroradius is performed, and the equations of motion are described in a form that clarifies the physics interpretation. Issues associated with the gauge transformation and the link to external perturbed fields obtained from wave propagation and MHD codes is addressed in Sec. IV. Finally, the summary and conclusions appear in Sec. V.

\section{LAGRANGIAN DETERMINATION OF CANONICAL VARIABLES}

The equilibrium magnetic field in the covariant representation in Boozer coordinates ${ }^{1}$ for anisotropic plasmas is

$$
\sigma \boldsymbol{B}=J(s) \boldsymbol{\nabla} \theta-I(s) \boldsymbol{\nabla} \phi+\sigma B_{s} \nabla s
$$

and in the contravariant representation is

$$
\begin{aligned}
\boldsymbol{B} & =\boldsymbol{\nabla} \phi \times \nabla \psi+q(\psi) \nabla \psi \times \nabla \theta=\nabla \alpha \times \nabla \psi \\
& =-\nabla \times(\psi \nabla \alpha),
\end{aligned}
$$

where $\theta$ and $\phi$ are the Boozer poloidal and toroidal angles, respectively, $\psi$ is the poloidal magnetic flux function, $I$ is the poloidal current flux function, $J$ is the toroidal current flux function, $q$ is the inverse rotational transform (safety factor), $B_{\psi} \equiv \sqrt{g} \boldsymbol{B} \cdot \boldsymbol{\nabla} \theta \times \boldsymbol{\nabla} \phi, \sqrt{g}$ is the Jacobian, $\alpha \equiv \phi-q(\psi) \theta$, and $\sigma=1-\mu_{0}\left(p_{\|}-p_{\perp}\right) / B^{2}$ is the anisotropy parameter when the parallel pressure $p_{\|}$is different from $p_{\perp}$ 
(perpendicular pressure). $\sigma>0$ indicates the firehose stability criterion. ${ }^{18}$ The full potential $\mathbf{A}$ can be written in the form

$$
\begin{aligned}
\boldsymbol{A}= & \boldsymbol{A}_{e}+\delta \boldsymbol{A}=-\psi \nabla \alpha+\delta A_{\phi} \boldsymbol{\nabla} \phi+\delta A_{\theta} \nabla \theta+\delta A_{\psi} \nabla \psi \\
= & U(\psi, \theta, \phi, t) \nabla \psi-\left[\psi-I(\psi) W_{\alpha}(\psi, \theta, \phi, t)\right] \nabla \alpha \\
& +V(\psi, \theta, \phi, t) \sigma \boldsymbol{B},
\end{aligned}
$$

where we define

$$
\begin{gathered}
V(\psi, \theta, \phi, t) \equiv \frac{\delta A_{\theta}(\psi, \theta, \phi, t)+q(\psi) \delta A_{\phi}(\psi, \theta, \phi, t)}{J(\psi)-q(\psi) I(\psi)}, \\
W_{\alpha}(\psi, \theta, \phi, t) \equiv \frac{\delta A_{\theta}(\psi, \theta, \phi, t)+[J(\psi) / I(\psi)] \delta A_{\phi}(\psi, \theta, \phi, t)}{J(\psi)-q(\psi) I(\psi)}, \\
U(\psi, \theta, \phi, t) \equiv \delta A_{\psi}(\psi, \theta, \phi, t) \\
-\frac{\left[\sigma B_{\psi}-\frac{d q}{d \psi} I(\psi) \theta\right] \delta A_{\theta}+\left[q(\psi) \sigma B_{\psi}-\frac{d q}{d \psi} J(\psi) \theta\right] \delta A_{\phi}}{J(\psi)-q(\psi) I(\psi)} .
\end{gathered}
$$

The momentum in the drift approximation is

$$
\boldsymbol{P}=P_{\|} \frac{\boldsymbol{B}}{B}+e \boldsymbol{A}
$$

where $e$ is the electronic charge of a particle. It is useful to define parallel and effective parallel gyroradii

$$
\begin{gathered}
\rho_{\|} \equiv P_{\|} /(e \sigma B), \\
\rho_{c} \equiv \rho_{\|}+V, \\
\rho_{w} \equiv \rho_{c}-W_{\alpha}=\rho_{\|}-\delta A_{\phi} / I(\psi) .
\end{gathered}
$$

The form $\rho_{c}$ is used in the formulation where only finite $\delta A_{\|}$is retained. ${ }^{2,11}$ The Lagrangian for the guiding center motion is

$$
\begin{aligned}
\mathcal{L} d t & =\boldsymbol{P} \cdot \mathbf{d} \mathbf{x}-\mathcal{H} d t \\
& =e\left(\rho_{\|} \sigma \boldsymbol{B}+\boldsymbol{A}\right) \cdot \mathbf{d x}-\mathcal{H} d t
\end{aligned}
$$

and the vector distance element

$$
\mathbf{d x}=\sqrt{g} \nabla \psi \times \nabla \theta d \alpha+\sqrt{g} \nabla \alpha \times \nabla \psi d \theta+\sqrt{g} \nabla \theta \times \nabla \alpha d \psi .
$$

Using $\phi=\alpha+q(\psi) \theta$, we express $\sigma \mathbf{B}$ as ${ }^{10,11}$

$$
\begin{gathered}
\sigma \boldsymbol{B}=I(\psi) \Delta(\psi, \theta) \nabla \psi+h(\psi) \nabla \theta-I(\psi) \nabla \alpha, \\
\Delta(\psi, \theta) \equiv \frac{\sigma B_{\psi}}{I(\psi)}-\frac{d q}{d \psi} \theta \\
h(\psi) \equiv J(\psi)-q(\psi) I(\psi) .
\end{gathered}
$$

Substituting this in the Lagrangian, we get

$$
\begin{aligned}
\frac{\mathcal{L} d t}{e}= & -\left[\psi+\rho_{w} I(\psi)\right] d \alpha+\rho_{c} h(\psi) d \theta \\
& +\left[\rho_{c} I(\psi) \Delta(\psi, \theta)+U(\psi, \theta, \phi, t)\right] d \psi-\frac{\mathcal{H} d t}{e} .
\end{aligned}
$$

This form of the Lagrangian does not satisfy canonical properties due to the finite amplitude of the term multiplying $d \psi,{ }^{3}$ which requires $\mathcal{L} d t=\sum_{i} p_{i} d q_{i}-\mathcal{H} d t$ ( $p_{i}$ are the canonical momenta and $q_{i}$ are the angular momenta).
The process of eliminating the $d \psi$ term entails defining the parameters ${ }^{10,11}$

$$
\begin{gathered}
\alpha_{c} \equiv-\alpha+\lambda\left(\psi, \theta, P_{\alpha}\right), \\
\lambda\left(\psi, \theta, P_{\alpha}\right) \equiv \int_{P_{\alpha}}^{\psi} \Delta(w, \theta) d w, \\
\mathcal{S} \equiv \int_{P_{\alpha}}^{\psi} w \Delta(w, \theta) d w,
\end{gathered}
$$

where $P_{\alpha}$ is the momentum associated with the $\alpha$ variable. As the Lagrangian is invariant to the addition or subtraction of a full differential, we can write

$$
\begin{aligned}
\frac{\mathcal{L} d t}{e}-d \mathcal{S}= & {\left[\rho_{c} h(\psi)-P_{\alpha} \frac{\partial \lambda}{\partial \theta}+\left.\int_{P_{\alpha}}^{\psi} w \frac{\partial \Delta}{\partial \theta}\right|_{w} d w\right] d \theta } \\
& +\left[\psi+\rho_{w} I(\psi)\right] d \alpha_{c}+[U(\psi, \theta, \phi, t) \\
& \left.+I(\psi) \Delta(\psi, \theta) W_{\alpha}(\psi, \theta, \phi, t)\right] d \psi-\frac{\mathcal{H} d t}{e} .
\end{aligned}
$$

This system remains noncanonical, but the $d \psi$ term now involves only perturbed vector potential projections. Without loss of generality, we can impose the gauge condition

$$
U(\psi, \theta, \phi, t)+I(\psi) \Delta(\psi, \theta) W_{\alpha}(\psi, \theta, \phi, t)=0 .
$$

Substituting for $U, W_{\alpha}$, and $\Delta$ simplifies the gauge condition to

$$
\delta A_{\psi}(\psi, \theta, \phi, t)=-\frac{\sigma B_{\psi}}{I(\psi)} \delta A_{\phi}(\psi, \theta, \phi, t) .
$$

The imposition of this gauge condition renders the Boozer coordinate frame canonical for guiding center drift motion with arbitrary perturbed electrostatic and electromagnetic fields in axisymmetric tokamak geometry.

The poloidal canonical angular momentum is

$$
P_{\theta}=\rho_{c} h(\psi)-\left.P_{\alpha} \frac{\partial \lambda}{\partial \theta}\right|_{\psi, P_{\alpha}}+\left.\int_{P_{\alpha}}^{\psi} w \frac{\partial \Delta}{\partial \theta}\right|_{w} d w .
$$

To proceed further, we introduce the identity ${ }^{10}$

$$
\frac{\partial \Delta(\psi, \theta)}{\partial \theta} \equiv \frac{\partial Q(\psi, \theta)}{\partial \psi}
$$

Then, the components of the canonical momentum are

$$
\begin{aligned}
P_{\theta} & =\rho_{c} h(\psi)+\left(\psi-P_{\alpha}\right) Q(\psi, \theta)-\int_{P_{\alpha}}^{\psi} Q(w, \theta) d w \\
& =\rho_{w}[h(\psi)-I(\psi) Q(\psi, \theta)]-\int_{P_{\alpha}}^{\psi} Q(w, \theta) d w+W(\psi, \theta, \phi, t),
\end{aligned}
$$

and

$$
P_{\alpha}=\psi+\rho_{w} I(\psi),
$$

where $W(\psi, \theta, \phi, t) \equiv h(\psi) W_{\alpha}(\psi, \theta, \phi, t)$. The canonical momenta are thus specified. 


\section{THE HAMILTONIAN FORMALISM}

The Hamiltonian for the guiding center drift motion is

$$
\frac{\mathcal{H}}{e}=\frac{e \rho_{\|}^{2} \sigma^{2} B^{2}}{2 m}+\frac{\mu B}{e}+\Phi_{E}=\mathcal{H}_{e},
$$

where $m$ is the particle mass, $\mu$ is the magnetic moment, $\Phi_{E}$ is the electrostatic potential, and the resulting equations of motion become

$$
\begin{gathered}
\frac{d P_{\theta}}{d t}=-\left.\frac{\partial \mathcal{H}_{e}}{\partial \theta}\right|_{P_{\theta}, P_{\alpha}, \alpha_{c}, t} \quad ; \quad \frac{d \theta}{d t}=\left.\frac{\partial \mathcal{H}_{e}}{\partial P_{\theta}}\right|_{P_{\alpha}, \theta, \alpha_{c}, t}, \\
\frac{d P_{\alpha}}{d t}=-\left.\frac{\partial \mathcal{H}_{e}}{\partial \alpha_{c}}\right|_{P_{\theta}, P_{\alpha}, \theta, t} \quad ; \quad \frac{d \alpha_{c}}{d t}=\left.\frac{\partial \mathcal{H}_{e}}{\partial P_{\alpha}}\right|_{P_{\theta}, \theta, \alpha_{c}, t},
\end{gathered}
$$

where we have dropped the subscript $c$ from $P_{\alpha}$ and the notation $\left.\right|_{\alpha, \beta, \gamma}$ denotes that the variables $\alpha, \beta$, and $\gamma$ are held fixed in the evaluation of the partial derivative. Expanding the Hamiltonian, invoking the relations presented in Appendix A yields the canonical equations of motion

$$
\begin{gathered}
\frac{d \theta}{d t}=\frac{e \sigma^{2} B^{2}}{m D} \rho_{\|}\left(1+\rho_{w} \frac{d I}{d \psi}\right)-\frac{1}{D}\left[I(\psi) D_{\psi}+\sigma B_{\psi} D_{\phi}\right] \\
\frac{d P_{\alpha}}{d t}=D_{\phi}-\left.\frac{\partial W}{\partial \phi}\right|_{\psi, \theta, t} \frac{d \theta}{d t}, \\
\frac{d P_{\theta}}{d t}=-D_{\theta}-\left[\left.\rho_{w} I(\psi) \frac{\partial Q}{\partial \theta}\right|_{\psi}+\left.\int_{P_{\alpha}}^{\psi} \frac{\partial Q}{\partial \theta}\right|_{w} d w-\left.\frac{\partial W}{\partial \theta}\right|_{\psi, \phi, t}\right] \frac{d \theta}{d t} \\
-\left[q(\psi)+Q(\psi, \theta)-Q\left(P_{\alpha}, \theta\right)\right]\left(D_{\phi}-\left.\frac{d \theta}{d t} \frac{\partial W}{\partial \phi}\right|_{\psi, \theta, t}\right), \\
\frac{d \alpha_{c}}{d t}= \\
\frac{e \sigma^{2} B^{2}}{m I(\psi)} \rho_{\|}-D_{\phi} \Delta\left(P_{\alpha}, \theta\right)+[q(\psi)+Q(\psi, \theta) \\
\left.-Q\left(P_{\alpha}, \theta\right)-\frac{J(\psi)}{I(\psi)}+\left.\Delta\left(P_{\alpha}, \theta\right) \frac{\partial W}{\partial \phi}\right|_{\psi, \theta, t}\right] \frac{d \theta}{d t},
\end{gathered}
$$

where the terms $D_{v}$ are written out in Appendix B and

$$
\begin{aligned}
D \equiv & J(\psi)-q(\psi) I(\psi) \\
& +\rho_{w}\left[J(\psi) \frac{d I}{d \psi}-I(\psi) \frac{d J}{d \psi}-\left.I(\psi) \frac{\partial\left(\sigma B_{\psi}\right)}{\partial \theta}\right|_{\psi}\right] \\
& -\left.I(\psi) \frac{\partial W}{\partial \psi}\right|_{\theta, \phi, t}-\left.\sigma B_{\psi} \frac{\partial W}{\partial \phi}\right|_{\psi, \theta, t} .
\end{aligned}
$$

The equations for the canonical momenta are really only useful for the application of symplectic integration schemes. It is physically more intuitive to follow the guiding center particle radial position $s[0 \leq s \leq 1$ in the plasma with $\psi=\psi(s)]$ and its parallel gyroradius $\rho_{\| \mid}{ }^{2,6,7,11}$ Invoking the algebraic manipulations described in Ref. 11 and the transformation to SI units prescribed in Appendix C, we obtain

$$
\begin{gathered}
\frac{d s}{d t}=\frac{\mu_{0} \mathcal{J}(s)}{D_{v}} D_{\phi}+\frac{\mu_{0} \mathcal{I}(s)}{D_{v}} D_{\theta}-\left.\frac{e \sigma^{2} B^{2}}{m D_{v}} \rho_{\|} \frac{\partial W}{\partial \phi}\right|_{s, \theta, t}, \\
\frac{d \theta}{d t}=\frac{e \sigma^{2} B^{2}}{m D_{v}} \rho_{\|}\left[\psi^{\prime}(s)+\rho_{w} \mu_{0} \mathcal{I}^{\prime}(s)\right]-\frac{\mu_{0} \mathcal{I}(s)}{D_{v}} D_{s}-\frac{\sigma B_{s}}{D_{v}} D_{\phi},
\end{gathered}
$$

$$
\begin{aligned}
& \frac{d \phi}{d t}=\frac{e \sigma^{2} B^{2}}{m D_{v}} \rho_{\|}\left[\Phi^{\prime}(s)+\rho_{w} \mu_{0} \mathcal{J}^{\prime}(s)-\left.\rho_{w} \frac{\partial\left(\sigma B_{s}\right)}{\partial \theta}\right|_{s}+\left.\frac{\partial W}{\partial s}\right|_{\theta, \phi, t}\right] \\
& -\frac{\mu_{0} \mathcal{J}(s)}{D_{v}} D_{s}+\frac{\sigma B_{s}}{D_{v}} D_{\theta}, \\
& \frac{d \rho_{\|}}{d t}=-\left.\frac{\partial T}{\partial t}\right|_{s, \theta, \phi}+\frac{1}{D_{v}}\left[\left.\mu_{0} \mathcal{I}(s) \frac{\partial T}{\partial \theta}\right|_{s, \phi, t}+\left.\mu_{0} \mathcal{J}(s) \frac{\partial T}{\partial \phi}\right|_{s, \theta, t}\right. \\
& \left.+\left.\frac{\partial W}{\partial \phi}\right|_{s, \theta, t}\right] D_{s_{2}}-\frac{1}{D_{v}}\left[\psi^{\prime}(s)+\rho_{w} \mu_{0} \mathcal{I}^{\prime}(s)\right. \\
& \left.+\left.\mu_{0} \mathcal{I}(s) \frac{\partial T}{\partial s}\right|_{\theta, \phi, t}+\left.\sigma B_{s} \frac{\partial T}{\partial \phi}\right|_{s, \theta, t}\right] D_{\theta_{2}} \\
& -\frac{1}{D_{v}}\left[\Phi^{\prime}(s)+\rho_{w} \mu_{0} \mathcal{J}^{\prime}(s)-\left.\rho_{w} \frac{\partial\left(\sigma B_{s}\right)}{\partial \theta}\right|_{s}\right. \\
& \left.+\left.\frac{\partial W}{\partial s}\right|_{\theta, \phi, t}+\left.\mu_{0} \mathcal{J}(s) \frac{\partial T}{\partial s}\right|_{\theta, \phi, t}-\left.\sigma B_{s} \frac{\partial T}{\partial \theta}\right|_{s, \phi, t}\right] D_{\phi_{2}} \text {. }
\end{aligned}
$$

The finite $\delta \mathbf{A}_{\perp}$ contributions appear through the derivatives of $W$. The forms presented in these equations recover the finite $\delta A_{\|}$model (Eqs. (43)-(46) described in Ref. 11) by taking $W \Rightarrow 0$, replacing $\rho_{w}$ with $\rho_{c}$ and $T$ with $V$. The terms involving $\sigma B_{s}$ are neglected in most other formulations of the guiding center drift problem. In this work, we retain all terms $O\left(\beta \rho_{\| \mid}\right)$. Expanding $W(s, \theta, \phi, t)=\delta A_{\theta}+[\mathcal{J}(s) / \mathcal{I}(s)] \delta A_{\phi}$ and $T(s, \theta, \phi, t)=-\delta A_{\phi} /\left[\mu_{0} \mathcal{I}(s)\right]$, we can alternatively write the guiding center particle drift orbit equations of motion as

$$
\begin{aligned}
& \frac{d s}{d t}=\frac{\mu_{0} \mathcal{I}(s)}{D_{v}}\left(\frac{\mu}{e}+\frac{e \sigma \tau B}{m} \rho_{\|}^{2}\right) \frac{\partial B}{\partial \theta} \\
& +\frac{1}{D_{v}}\left[\left.\mu_{0} \mathcal{I}(s) \frac{\partial \Phi_{E}}{\partial \theta}\right|_{s, \phi, t}+\left.\mu_{0} \mathcal{J}(s) \frac{\partial \Phi_{E}}{\partial \phi}\right|_{s, \theta, t}\right] \\
& +\frac{e \sigma^{2} B^{2}}{m D_{v}} \rho_{\|}\left[\left.\frac{\partial\left(\delta A_{\phi}\right)}{\partial \theta}\right|_{s, \phi, t}-\left.\frac{\partial\left(\delta A_{\theta}\right)}{\partial \phi}\right|_{s, \theta, t}\right] \text {, } \\
& \frac{d \theta}{d t}=-\frac{\mu_{0} \mathcal{I}(s)}{D_{v}}\left[\left(\frac{\mu}{e}+\frac{e \sigma \tau B}{m} \rho_{\|}^{2}\right) \frac{\partial B}{\partial s}+\left.\frac{e \sigma B^{2}}{m} \rho_{\|}^{2} \frac{\partial \sigma}{\partial s}\right|_{B}\right] \\
& +\frac{e \sigma^{2} B^{2}}{m D_{v}} \rho_{\|}\left[\psi^{\prime}(s)+\mu_{0} \mathcal{I}^{\prime}(s) \rho_{\|}\right]-\frac{1}{D_{v}}\left[\left.\mu_{0} \mathcal{I}(s) \frac{\partial \Phi_{E}}{\partial s}\right|_{\theta, \phi, t}\right. \\
& \left.+\left.\sigma B_{s} \frac{\partial \Phi_{E}}{\partial \phi}\right|_{s, \theta, t}\right]-\frac{e \sigma^{2} B^{2}}{m D_{v}} \rho_{\|}\left[\left.\frac{\partial\left(\delta A_{\phi}\right)}{\partial s}\right|_{\theta, \phi, t}\right. \\
& \left.+\left.\frac{\sigma B_{s}}{\mu_{0} \mathcal{I}(s)} \frac{\partial\left(\delta A_{\phi}\right)}{\partial \phi}\right|_{s, \theta, t}\right] \text {, } \\
& \frac{d \phi}{d t}=-\frac{\mu_{0} \mathcal{J}(s)}{D_{v}}\left[\left(\frac{\mu}{e}+\frac{e \sigma \tau B}{m} \rho_{\|}^{2}\right)\left(\frac{\partial B}{\partial s}-\frac{\sigma B_{s}}{\mu_{0} \mathcal{J}(s)} \frac{\partial B}{\partial \theta}\right)\right. \\
& \left.+\left.\frac{e \sigma B^{2}}{m} \rho_{\|}^{2} \frac{\partial \sigma}{\partial s}\right|_{B}\right]+\frac{e \sigma^{2} B^{2}}{m D_{v}} \rho_{\|}\left[\Phi^{\prime}(s)+\rho_{\|}\left(\mu_{0} \mathcal{J}^{\prime}(s)\right.\right. \\
& \left.\left.-\frac{\partial\left(\sigma B_{s}\right)}{\partial \theta}\right)\right]-\frac{\mu_{0} \mathcal{J}(s)}{D}\left[\left.\frac{\partial \Phi_{E}}{\partial s}\right|_{\theta, \phi, t}-\left.\frac{\sigma B_{s}}{\mu_{0} \mathcal{J}(s)} \frac{\partial \Phi_{E}}{\partial \theta}\right|_{s, \phi, t}\right] \\
& +\frac{e \sigma^{2} B^{2}}{m D_{v}} \rho_{\|}\left[\left.\frac{\partial\left(\delta A_{\theta}\right)}{\partial s}\right|_{\theta, \phi, t}+\frac{1}{\mu_{0} \mathcal{I}(s)}\left(\left.\sigma B_{s} \frac{\partial\left(\delta A_{\phi}\right)}{\partial \theta}\right|_{s, \phi, t}\right.\right. \\
& \left.\left.+\frac{\partial\left(\sigma B_{s}\right)}{\partial \theta} \delta A_{\phi}\right)\right]
\end{aligned}
$$


where $\tau$ is the mirror stability criterion parameter. ${ }^{8,18}$ For the parallel gyroradius, we have

$$
\begin{aligned}
& \frac{d \rho_{\|}}{d t}=-\frac{1}{D_{v}}\left(\frac{\mu}{e}+\frac{e \sigma \tau B}{m} \rho_{\|}^{2}\right)\left[\psi^{\prime}(s)+\mu_{0} \mathcal{I}^{\prime}(s) \rho_{\|}\right] \frac{\partial B}{\partial \theta} \\
& -\left.\frac{1}{D_{v}}\left[\psi^{\prime}(s)+\mu_{0} \mathcal{I}^{\prime}(s) \rho_{\|}\right] \frac{\partial \Phi_{E}}{\partial \theta}\right|_{s, \phi, t} \\
& -\left.\frac{1}{D_{v}}\left[\Phi^{\prime}(s)+\rho_{\|}\left(\mu_{0} \mathcal{J}^{\prime}(s)-\frac{\partial\left(\sigma B_{s}\right)}{\partial \theta}\right)\right] \frac{\partial \Phi_{E}}{\partial \phi}\right|_{s, \theta, t} \\
& +\frac{1}{D_{v}}\left(\frac{\mu}{e}+\frac{e \sigma \tau B}{m} \rho_{\|}^{2}\right) \frac{\partial B}{\partial \theta}\left[\left.\frac{\partial\left(\delta A_{\phi}\right)}{\partial s}\right|_{\theta, \phi, t}\right. \\
& \left.+\left.\frac{\sigma B_{s}}{\mu_{0} \mathcal{I}(s)} \frac{\partial\left(\delta A_{\phi}\right)}{\partial \phi}\right|_{s, \theta, t}\right]+\left.\frac{1}{\mu_{0} \mathcal{I}(s)} \frac{\partial\left(\delta A_{\phi}\right)}{\partial t}\right|_{s, \theta, \phi} \\
& -\frac{1}{D_{v}}\left[\left(\frac{\mu}{e}+\frac{e \sigma \tau B}{m} \rho_{\|}^{2}\right) \frac{\partial B}{\partial s}+\left.\frac{e \sigma B^{2}}{m} \rho_{\|}^{2} \frac{\partial \sigma}{\partial s}\right|_{B}\right] \\
& \times\left[\left.\frac{\partial\left(\delta A_{\phi}\right)}{\partial \theta}\right|_{s, \phi, t}-\left.\frac{\partial\left(\delta A_{\theta}\right)}{\partial \phi}\right|_{s, \theta, t}\right]-\frac{1}{D_{v}}\left[\left.\frac{\partial\left(\delta A_{\phi}\right)}{\partial \theta}\right|_{s, \phi, t}\right. \\
& \left.-\left.\frac{\partial\left(\delta A_{\theta}\right)}{\partial \phi}\right|_{s, \theta, t}\right]\left.\frac{\partial \Phi_{E}}{\partial s}\right|_{\theta, \phi, t}+\frac{1}{D_{v}}\left[\left.\frac{\partial\left(\delta A_{\phi}\right)}{\partial s}\right|_{\theta, \phi, t}\right. \\
& \left.+\left.\frac{\sigma B_{s}}{\mu_{0} \mathcal{I}(s)} \frac{\partial\left(\delta A_{\phi}\right)}{\partial \phi}\right|_{s, \theta, t}\right]\left.\frac{\partial \Phi_{E}}{\partial \theta}\right|_{s, \phi, t}-\frac{1}{D_{v}}\left[\left.\frac{\partial\left(\delta A_{\theta}\right)}{\partial s}\right|_{\theta, \phi, t}\right. \\
& \left.+\frac{1}{\mu_{0} \mathcal{I}(s)}\left(\left.\sigma B_{s} \frac{\partial\left(\delta A_{\phi}\right)}{\partial \theta}\right|_{s, \phi, t}+\frac{\partial\left(\sigma B_{s}\right)}{\partial \theta} \delta A_{\phi}\right)\right]\left.\frac{\partial \Phi_{E}}{\partial \phi}\right|_{s, \theta, t} .
\end{aligned}
$$

In the evolution of the radial particle positions Eq. (33), the first term corresponds to the particle interaction with the equilibrium fields, the second term is the interaction of the particle with the electrostatic perturbed field, and the last term deals with particle interaction with the electromagnetic perturbed field. For the evolution of the angular coordinates, the first two terms govern the motion of particles in the equilibrium field, the third term constitutes the particle interaction with the electrostatic perturbed field and the last term is its interaction with the electromagnetic perturbed field, respectively. With respect to the parallel gyroradius Eq. (36), the first term corresponds to motion in the equilibrium field, the second and third terms constitute interactions of particles with the electrostatic perturbed field, the fourth, fifth, and sixth terms are particle interactions with the perturbed electromagnetic field. The last three terms constitute wave-wave interactions. They are consequently nonlinear terms and describe the coupling of the perturbed electrostatic potential with perturbed electromagnetic field projections. Previously, the guiding center drift problem with full perturbed fields was investigated in 3D geometry. ${ }^{8}$ Although we limit ourselves to axisymmetric geometry in this work, we include the radial component of the equilibrium magnetic field in the covariant representation which was neglected in Ref. 8 .

\section{POTENTIAL STRUCTURE FOR FULL ELECTROMAGNETIC FIELDS}

The vector potential is only known up to the gradient of a scalar function. If we consider the output of a wave propagation code like LEMan, ${ }^{19}$ which computes low frequency waves and employs the Coulomb gauge, we must make then the identification

$$
\delta A^{D}+\nabla G=\delta A^{E W},
$$

where the superscript $D$ stands for the form of the vector potential required for the drift orbit description and the superscript EW denotes the external wave field calculated with a solver like LEMan. The radial, toroidal, and poloidal covariant projections (for example, $\sqrt{g} \nabla \theta \times \nabla \phi$ ) invoking the gauge condition $\delta A_{s}=-\sigma B_{s} \delta A_{\phi} /\left[\mu_{0} \mathcal{I}(s)\right]$ yield

$$
\begin{aligned}
\mu_{0} \mathcal{I}(s) \frac{\partial G}{\partial s}+\sigma B_{s} \frac{\partial G}{\partial \phi} & =\mu_{0} \mathcal{I}(s) \delta A_{s}^{E W}+\sigma B_{s} \delta A_{\phi}^{E W}, \\
\delta A_{\phi}^{D} & =\delta A_{\phi}^{E W}-\frac{\partial G}{\partial \phi} \\
\delta A_{\theta}^{D} & =\delta A_{\theta}^{E W}-\frac{\partial G}{\partial \theta} .
\end{aligned}
$$

Thus, we first would need to solve Eq. (38) for the scalar function $G$. Subsequently, we compute $\delta A_{\phi}^{D}$ and $\delta A_{\theta}^{D}$. To determine the electrostatic potential $\Phi_{E}$, we invoke Faraday's Law from which we obtain either

$$
\frac{\partial \Phi_{E}^{D}}{\partial \phi}=\frac{\partial \Phi_{E}^{E W}}{\partial \phi}+\frac{\partial \delta A_{\phi}^{E W}}{\partial t}-\frac{\partial \delta A_{\phi}^{D}}{\partial t},
$$

or

$$
\frac{\partial \Phi_{E}^{D}}{\partial \theta}=\frac{\partial \Phi_{E}^{E W}}{\partial \theta}+\frac{\partial \delta A_{\theta}^{E W}}{\partial t}-\frac{\partial \delta A_{\theta}^{D}}{\partial t}
$$

Typically a Fourier method would be applied; hence, Eq. (41) would be used to solve for all toroidal mode number $n \neq 0$ amplitudes of $\Phi_{E}^{D}$, while Eq. (42) would be applied for the remaining $n=0$ and poloidal mode number $m \neq 0$ terms.

For the evolution of the guiding center particle orbits in MHD instability structures, then the MHD vector potential is $\delta \boldsymbol{A}^{M H D}=\xi \times \boldsymbol{B}$ (where $\xi$ is the perturbed displacement vector) and the electrostatic potential $\Phi_{E}^{M H D}=0$. Typically in codes like TERPSICHORE, ${ }^{20}$ we express

$$
\boldsymbol{\xi}=\sqrt{g} \xi^{s} \nabla \theta \times \nabla \phi+\frac{\boldsymbol{B} \times \nabla s}{B^{2}} \eta+\frac{\xi_{\|}}{B} \boldsymbol{B} .
$$

The vector potential components in the MHD model reduce to

$$
\delta A_{s}^{M H D}=\eta ; \quad \delta A_{\phi}^{M H D}=\psi^{\prime}(s) \xi^{s} ; \quad \delta A_{\theta}^{M H D}=-\Phi^{\prime}(s) \xi^{s} .
$$

After applying Faraday's Law [Eqs. (41) or (42)], replacing the label EW with MHD and considering the time derivative of projections of Eq. (37), we get the relation

$$
\Phi_{E}^{D}=\frac{\partial G}{\partial t}
$$

Then, the radial, toroidal, and poloidal components of the vector potential for the drift motion become 


$$
\begin{gathered}
\mu_{0} \mathcal{I}(s) \frac{\partial \Phi_{E}^{D}}{\partial s}+\sigma B_{s} \frac{\partial \Phi_{E}^{D}}{\partial \phi}=\mu_{0} \mathcal{I}(s) \frac{\partial \eta}{\partial t}+\sigma B_{s} \psi^{\prime}(s) \frac{\partial \xi^{s}}{\partial t} \\
\frac{\partial \delta A_{\phi}^{D}}{\partial t}=\psi^{\prime}(s) \frac{\partial \xi^{s}}{\partial t}-\frac{\partial \Phi_{E}^{D}}{\partial \phi} \\
\frac{\partial \delta A_{\theta}^{D}}{\partial t}=-\Phi^{\prime}(s) \frac{\partial \xi^{s}}{\partial t}-\frac{\partial \Phi_{E}^{D}}{\partial \theta}
\end{gathered}
$$

In this case, we solve first for $\Phi_{E}^{D}(s, \theta, \phi, t)$ and subsequently obtain $\delta A_{\phi}^{D}$ and $\delta A_{\theta}^{D}$.

\section{SUMMARY AND CONCLUSIONS}

The Boozer coordinate frame remains canonical for the description of a Hamiltonian/Lagrangian theory of guiding center particle drift motion that includes full static equilibrium, perturbed electrostatic and perturbed electromagnetic fields in axisymmetric tokamak geometry for plasma conditions that allow anisotropic pressure from auxiliary heated energetic particle species. We have thus extended the method developed by Wang ${ }^{10}$ and Cooper et al. ${ }^{11}$ to consider also the impact of finite $\delta \boldsymbol{A}_{\perp}$ on the orbit evolution. A critical step is then imposition of a gauge transformation for $\delta \boldsymbol{A}$ to guarantee that the Boozer coordinates retain canonical structure. The guiding center particle coordinate position evolution contains only equilibrium field effects and linear wave-particle interactions. Nonlinear wave-wave interactions from the perturbed fields consist of couplings between vector potential components with the electrostatic potential and impact only the evolution of the parallel gyroradius.

As the vector potential is only known to within the gradient of a scalar function $G$, we solve first for $G$ which depends on the radial and toroidal components of the external electromagnetic potential in the covariant representation for fields obtained from a wave propagation code. Once this is known, the perturbed electromagnetic field components $\delta A_{\phi}^{D}$ and $\delta A_{\theta}^{D}$ required for the drift motion can be evaluated and subsequently the application of Faraday's law yields the electrostatic potential. For fields obtained from a MHD stability code, the electrostatic potential is calculated first in terms of the binormal and radial components of the displacement vector $\xi$. This scalar potential depends mainly on the binormal component $\eta$ of $\xi$. For models that retain only finite $\delta A_{\|,},{ }^{2,6,7,11}$ the equivalence of the perturbed radial magnetic field $\delta \boldsymbol{B} \cdot \nabla s$ in the drift and external field representations of the potentials is invoked and this leads to a dependence of the electrostatic potential $\Phi_{E}$ on the radial component of $\xi$ for motion in MHD-like fields. This difference of the $\Phi_{E}$ dependence will be an important issue to address in future numerical simulations. Once $\Phi_{E}$ is determined, the electromagnetic potential components $\delta A_{\phi}^{D}$ and $\delta A_{\theta}^{D}$ can be linked to their MHD counterparts.

The equations we have presented are more clearly designed for drift kinetic analysis of magnetically confined plasmas. However, the applicability to gyrokinetic modeling can also be considered. The impact of full electromagnetic effects in a nonlinear gyrokinetic description of a plasma could be assessed with the guiding center drift orbit equations we have derived. For example, this would entail replacing Eqs. (26) and (27) in Ref. 21 with Eqs. (33)-(36) of this manuscript, after performing gyro-orbit averaging. The nonlinear terms in the $\rho_{\|}$Eq. (36) would constitute a drift nonlinearity (to second order in the perturbed field expansion), which is formally of the same order as the second order parallel velocity nonlinearity described in Ref. 21 .

\section{ACKNOWLEDGMENTS}

We appreciate numerous valuable discussions with Dr. Stephan Brunner. This research was partially sponsored by the Fonds National Suisse de la Recherche Scientifique and Euratom.

\section{APPENDIX A: DERIVATIVES WITH RESPECT TO THE CANONICAL VARIABLES}

The quantities $\psi, \rho_{w}$, and $\phi$ can be expressed as functions of the canonical variables. Hence, we derive the following relations

$$
\begin{aligned}
& \left.\frac{\partial \psi}{\partial P_{\theta}}\right|_{P_{\alpha}, \theta, \alpha_{c}}=-\frac{I(\psi)}{D}, \\
& \left.\frac{\partial \psi}{\partial P_{\alpha}}\right|_{P_{\theta}, \theta, \alpha_{c}}=-\frac{I(\psi)}{D}\left[q(\psi)+Q(\psi, \theta)-Q\left(P_{\alpha}, \theta\right)-\frac{J(\psi)}{I(\psi)}\right. \\
& \left.+\left.\Delta\left(P_{\alpha}, \theta\right) \frac{\partial W}{\partial \phi}\right|_{\psi, \theta, t}\right] \\
& \left.\frac{\partial \psi}{\partial \theta}\right|_{P_{\theta}, P_{\alpha}, \alpha_{c}}=-\frac{I(\psi)}{D}\left\{\left.\rho_{w} I(\psi) \frac{\partial Q}{\partial \theta}\right|_{\psi}+\left.\int_{P_{\alpha}}^{\psi} d \omega \frac{\partial Q}{\partial \theta}\right|_{\omega}-\left.\frac{\partial W}{\partial \theta}\right|_{\psi, \phi, t}\right. \\
& \left.-\left.\left[q(\psi)+Q(\psi, \theta)-Q\left(P_{\alpha}, \theta\right)\right] \frac{\partial W}{\partial \phi}\right|_{\psi, \theta, t}\right\} \text {, } \\
& \left.\frac{\partial \psi}{\partial \alpha_{c}}\right|_{P_{\theta}, P_{\alpha}, \theta}=-\left.\frac{I(\psi)}{D} \frac{\partial W}{\partial \phi}\right|_{\psi, \theta, t}, \\
& \left.\frac{\partial \rho_{w}}{\partial P_{\theta}}\right|_{P_{\alpha}, \theta, \alpha_{c}}=\frac{1}{D}\left(1+\rho_{w} \frac{d I}{d \psi}\right), \\
& \left.\frac{\partial \rho_{w}}{\partial P_{\alpha}}\right|_{P_{\theta}, \theta, \alpha_{c}}=-\left.\frac{1}{I(\psi)}\left(1+\rho_{w} \frac{d I}{d \psi}\right) \frac{\partial \psi}{\partial P_{\alpha}}\right|_{P_{\theta}, \theta, \alpha_{c}}+\frac{1}{I(\psi)}, \\
& \left.\frac{\partial \rho_{w}}{\partial \theta}\right|_{P_{\theta}, P_{\alpha}, \alpha_{c}}=-\left.\frac{1}{I(\psi)}\left(1+\rho_{w} \frac{d I}{d \psi}\right) \frac{\partial \psi}{\partial \theta}\right|_{P_{\theta}, P_{\alpha}, \alpha_{c}}, \\
& \left.\frac{\partial \rho_{w}}{\partial \alpha_{c}}\right|_{P_{\theta}, P_{\alpha}, \theta}=\left.\frac{1}{D}\left(1+\rho_{w} \frac{d I}{d \psi}\right) \frac{\partial W}{\partial \phi}\right|_{\psi, \theta, t} .
\end{aligned}
$$

Noting that $\phi=-\alpha_{c}+q(\psi) \theta+\lambda\left(\psi, \theta, P_{\alpha}\right)$, then

$$
\begin{gathered}
\left.\frac{\partial \phi}{\partial P_{\theta}}\right|_{P_{\alpha}, \theta, \alpha_{c}}=-\frac{\sigma B_{\psi}}{D}, \\
\left.\frac{\partial \phi}{\partial P_{\alpha}}\right|_{P_{\theta}, \theta, \alpha_{c}}=\left.\frac{\sigma B_{\psi}}{I(\psi)} \frac{\partial \psi}{\partial P_{\alpha}}\right|_{P_{\theta}, \theta, \alpha_{c}}-\Delta\left(P_{\alpha}, \theta\right), \\
\left.\frac{\partial \phi}{\partial \theta}\right|_{P_{\theta}, P_{\alpha}, \alpha_{c}}=q(\psi)+Q(\psi, \theta)-Q\left(P_{\alpha}, \theta\right)+\left.\frac{\sigma B_{\psi}}{I(\psi)} \frac{\partial \psi}{\partial \theta}\right|_{P_{\theta}, P_{\alpha}, \alpha_{c}}, \\
\left.\frac{\partial \phi}{\partial \alpha_{c}}\right|_{P_{\theta}, P_{\alpha}, \theta}=-1-\left.\frac{\sigma B_{\psi}}{D} \frac{\partial W}{\partial \phi}\right|_{\psi, \theta, t} .
\end{gathered}
$$




\section{APPENDIX B: DEFINITION OF $D_{v}$ TERMS}

The notation adopted in Ref. 11 is used. Thus

$$
\begin{gathered}
D_{\psi}=D_{\psi_{1}}+D_{\psi_{2}}, \\
D_{\theta}=D_{\theta_{1}}+D_{\theta_{2}}, \\
D_{\phi}=D_{\phi_{1}}+D_{\phi_{2}}, \\
D_{\psi_{1}}=-\left.\frac{e \sigma^{2} B^{2}}{m} \rho_{\|} \frac{\partial T}{\partial \psi}\right|_{\theta, \phi, t}, \\
D_{\psi_{2}}=\left(\frac{e \tau \sigma B}{m} \rho_{\|}^{2}+\frac{\mu}{e}\right) \frac{\partial B}{\partial \psi}+\left.\frac{e \sigma B^{2}}{m} \rho_{\|}^{2} \frac{\partial \sigma}{\partial \psi}\right|_{B}+\left.\frac{\partial \Phi_{E}}{\partial \psi}\right|_{\theta, \phi, t}, \\
D_{\theta_{1}}=-\left.\frac{e \sigma^{2} B^{2}}{m} \rho_{\|} \frac{\partial T}{\partial \theta}\right|_{\psi, \phi, t}, \\
D_{\theta_{2}}=\left(\frac{e \tau \sigma B}{m} \rho_{\|}^{2}+\frac{\mu}{e}\right) \frac{\partial B}{\partial \theta}+\left.\frac{\partial \Phi_{E}}{\partial \theta}\right|_{\psi, \phi, t}, \\
D_{\phi_{1}}=-\left.\frac{e \sigma^{2} B^{2}}{m} \rho_{\|} \frac{\partial T}{\partial \phi}\right|_{\psi, \theta, t}, \\
D_{\phi_{2}}=\left.\frac{\partial \Phi_{E}}{\partial \phi}\right|_{\psi, \theta, t},
\end{gathered}
$$

where $\tau$ is the mirror stability criterion parameter. ${ }^{15,18}$

\section{APPENDIX C: CONVERSION TO SI UNITS}

To convert the guiding center particle equations of motion to SI units and in the form applicable for implementation in the VENUS code, ${ }^{22}$ we identify $I(\psi) \Rightarrow \mu_{0} \mathcal{I}(s)$, $J(\psi) \Rightarrow \mu_{0} \mathcal{J}(s), \quad \sigma B_{\psi} \Rightarrow \sigma B_{s} / \psi^{\prime}(s), \quad D \Rightarrow D_{v} / \psi^{\prime}(s), \quad D_{\psi}$ $\Rightarrow D_{s} / \psi^{\prime}(s)$, and $d \gamma / d \psi \Rightarrow \gamma^{\prime}(s) / \psi^{\prime}(s)$ for any variable $\gamma$ that is a flux surface quantity. Note that the Jacobian becomes

$$
\sqrt{g}_{v}=\mu_{0}\left[\psi^{\prime}(s) \mathcal{J}(s)-\Phi^{\prime}(s) \mathcal{I}(s)\right] /\left(\sigma B^{2}\right),
$$

and $D_{v}$ is

$$
\begin{aligned}
D_{v}= & \sigma \sqrt{g}_{v} B^{2}+\mu_{0} \rho_{\|}\left[\mu_{0} \mathcal{J}(s) \mathcal{I}^{\prime}(s)-\mu_{0} \mathcal{I}(s) \mathcal{J}^{\prime}(s)\right. \\
& +\left.\mathcal{I}(s) \frac{\partial\left(\sigma B_{s}\right)}{\partial \theta}\right|_{s}-\left.\mu_{0} \mathcal{I} \frac{\partial\left(\delta A_{\theta}\right)}{\partial s}\right|_{\theta, \phi, t}-\left.\mu_{0} \mathcal{J} \frac{\partial\left(\delta A_{\phi}\right)}{\partial s}\right|_{\theta, \phi, t} \\
& -\sigma B_{s}\left[\left.\frac{\partial\left(\delta A_{\theta}\right)}{\partial \phi}\right|_{s, \theta, t}+\left.\frac{\mathcal{J}(s)}{\mathcal{I}(s)} \frac{\partial\left(\delta A_{\phi}\right)}{\partial \phi}\right|_{s, \theta, t}\right] \\
& -\left.\frac{\partial\left(\sigma B_{s}\right)}{\partial \theta}\right|_{s} \delta A_{\phi}(s, \theta, \phi, t) .
\end{aligned}
$$

${ }^{1}$ A. H. Boozer, Phys. Fluids 23, 904 (1980).

${ }^{2}$ R. B. White and M. S. Chance, Phys. Fluids 27, 2455 (1984).

${ }^{3}$ R. G. Littlejohn, Phys. Fluids 28, 2015 (1985).

${ }^{4}$ J. D. Meiss and R. D. Hazeltine, Phys. Fluids B2, 2563 (1990).

${ }^{5}$ W. A. Cooper, Plasma Phys. Controlled Fusion 39, 931 (1997).

${ }^{6}$ R. B. White, Phys. Fluids B2, 845 (1990).

${ }^{7}$ S. D. Pinches, L. C. Appel, J. Candy, S. E. Sharapov, H. L. Berk, D. Borba, B. N. Breizman, T. C. Hender, K. I. Hopcraft, G. T. A. Huysmans, and W. Kerner, Comput. Phys. Commun. 111, 133 (1998).

${ }^{8}$ W. A. Cooper, J. P. Graves, S. Brunner, and M. Yu. Isaev, Plasma Phys. Controlled Fusion 53, 24001 (2011).

${ }^{9}$ R. White and L. E. Zakharov, Phys. Plasmas 10, 573 (2003).

${ }^{10}$ S. Wang, Phys. Plasmas 13, 052506 (2006).

${ }^{11}$ G. A. Cooper, M. Jucker, W. A. Cooper, J. P. Graves, and M. Yu. Isaev, Phys. Plasmas 14, 102506 (2007).

${ }^{12}$ T. Yamaguchi, K. Y. Watanabe, S. Sakakibara, Y. Narushima, K. Narihara, T. Tokuzawa, K. Tanaka, I. Yamada, M. Osakabe, H. Yamada, K. Kawahata, K. Yamazaki, and LHD experimental group, Nucl. Fusion 45, L33 (2005).

${ }^{13}$ N. A. Madden and R. J. Hastie, Nucl. Fusion 34, 519 (1994).

${ }^{14}$ W. Zwingmann, L. G. Eriksson, and P. Stubberfield, Plasma Phys. Controlled Fusion 43, 1441 (2001).

${ }^{15}$ W. A. Cooper, S. P. Hirshman, T. Yamaguchi, Y. Narushima, S. Okamura, S. Sakakibara, C. Suzuki, K. Y. Watanabe, H. Yamada, and K. Yamazaki, Plasma Phys. Controlled Fusion 47, 561 (2005).

${ }^{16}$ W. A. Cooper, J. P. Graves, S. P. Hirshman, T. Yamaguchi, Y. Narushima, S. Okamura, S. Sakakibara, C. Suzuki, K. Y. Watanabe, H. Yamada, and K. Yamazaki, Nucl. Fusion 46, 683 (2006).

${ }^{17}$ V. D. Pustovitov, Plasma Phys. Controlled Fusion 52, 065001 (2010).

${ }^{18}$ H. Grad, Phys. Fluids 9, 498 (1966).

${ }^{19}$ P. Popovich, W. A. Cooper, and L. Villard, Comput. Phys. Commun. 175, 250 (2006).

${ }^{20}$ D. V. Anderson, W. A. Cooper, R. Gruber, S. Merazzi, and U. Schwenn, Int. J. Supercomput. Appl. 4, 34 (1990).

${ }^{21}$ A. Brizard, J. Plasma Phys. 41, 541 (1989).

${ }^{22}$ O. Fischer, W. A. Cooper, L. Villard, and M. Yu. Isaev, Nucl. Fusion 42, 817 (2002). 